\title{
Politique
}

Politique

\section{Où va le modèle suédois? État-providence et protection sociale de Lionel-Henri Groulx, Montréal/Paris, Presses de l'Université de Montréal/L'Harmattan, 1990, 151 p.}

\section{Pierre P. Tremblay}

Numéro 21, hiver 1992

URI : https://id.erudit.org/iderudit/040725ar

DOI : https://doi.org/10.7202/040725ar

Aller au sommaire du numéro

Éditeur(s)

Société québécoise de science politique

ISSN

0711-608X (imprimé)

1918-6584 (numérique)

Découvrir la revue

Citer ce compte rendu

Tremblay, P. P. (1992). Compte rendu de [Où va le modèle suédois?

État-providence et protection sociale de Lionel-Henri Groulx, Montréal/Paris, Presses de l'Université de Montréal/L'Harmattan, 1990, 151 p.] Politique, (21),

190-192. https://doi.org/10.7202/040725ar d'utilisation que vous pouvez consulter en ligne.

https://apropos.erudit.org/fr/usagers/politique-dutilisation/ 
Oi va le modèle suédois? État-providence et protection sociale

de Lionel-Henri GROULX, Montréal/Paris, Presses de I'Université de Montréal/L'Harmattan, 1990, 151 p.

Le livre de Lionel-Henri Groulx entend démontrer que les Suédois ont entrepris de sauver leur modèle de l'État-providence en lui injectant une forte dose de valeurs et d'exigences propres au marché. Toutefois, selon l'auteur, ce modele rafraîchi au vent du néo-libéralisme introduira des distorsions dans la satisfaction des besoins par rapport aux principes d'égalité et de redistribution sociales. Voilà, presque en version intégrale, la thèse de l'ouvrage.

Groulx propose au lecteur un texte articulé autour de trois volets du modèle suédois : la politique de l'emploi, les services sociaux et de santé ainsi que la sécurité du revenu. Dans le premier chapitre, on apprend (p. 15) que la politique de l'emploi est conçue selon les caractéristiques de la protection sociale de ce pays, à savoir la «décommodification" et l'universalité. La première caractéristique signifie qu'un bien ou un service n'obéit pas aux lois du marché et du profit. C'est justement sur la mutation de cette qualité du système que l'auteur élabore sa description de la politique. II termine le chapitre en affirmant que de nouvelles règles et de nouvelles normes plus libérales valorisant la flexibilité, l'efficacité et la compétitivité faussent le principe de la «décommodification". On sent, au cours de cette première séquence d'analyse, que Groulx $n$ 'est pas à l'aise en science économique. Sa démarche est boiteuse, et l'utilisation des données proposées au lecteur est plutôt lacunaire. À titre d'exemple, le tableau 1.4 traitant de l'indemnisation du système d'assurance-chômage est incomplet tandis que le tableau 1.5 (Dépenses pour la politique de l'emploi 19741985) fait l'objet d'un commentaire de quatre lignes. C'est peu pour un programme de dépenses important.

Le second chapitre consacré aux services sociaux et de santé est nettement meilleur. L'exposé y est plus étoffé et plus vivant. Groulx traite des services sociaux, des services 
de garde, des services aux personnes ågées et aux personnes handicapées ainsi que des services de santé. On y apprend que les Suédois se posent les mêmes questions que les Québécois. La privatisation des services sociaux, le ticket modérateur et la centralisation des services de santé vers les hôpitaux sont autant de débats dont la conclusion n'est pas encore évidente. L'auteur apporte à ce chapitre des éléments d'analyse et d'évaluation intéressants. Ainsi, à la page 60 , il écrit : $\kappa$ Tout se passe comme si le modèle suédois se butait aux conséquences engendrées par son développement [...] qu'il obéissait à des processus ou règles de fonctionnement de plus en plus indépendantes des modes de solidarité sociale qui ont suscité leur émergencen. Groulx remet en question le corporatisme des professionnels du secteur de la santé et des services sociaux et en esquisse les effets pervers pour l'avenir de la solidarité sociale.

Le troisième chapitre s'attaque à la sécurité du revenu. Cette politique donne à la Suède un rang enviable sur l'échelle mondiale de la richesse. Groulx affirme, statistiques à l'appui, que, grâce aux transferts, il n'y a que $5 \%$ de la population suédoise vivant sous le seuil de la pauvreté. Ce qui est une performance supérieure à celles du Canada, de l'Allemagne de l'Ouest, de la Grande-Bretagne, des États-Unis, etc. Par contre, sans les dépenses de transferts, la Suède verrait la proportion de pauvres grimper à $41 \%$. La réforme possible de la protection du revenu susciterait le passage d'une logique de prestations uniformes et universelles a une logique de prestations différentielles au risque de renforcer la logique inégalitaire du marché (p. 88). L'auteur constate là un danger pour le principe de l'égalité, ce qui accélère de plus l'érosion du modèle suédois de protection sociale.

Dans un dernier chapitre avant la conclusion, LionelHenri Groulx présente deux critiques du modèle : une critique néo-libérale et une critique sociocommunautaire. La première prétend qu'une modification de l'État-providence est impérative pour le développement de l'économie et elle conteste le principe de l'égalité. En contrepartie, la critique sociocommunautaire ne croit pas l'État capable d'assurer adéquatement la solidarité sociale. Toutes deux définissent l'État comme une entité essentiellement bureaucratique, coercitive 
et inefficace. L'auteur associe ces deux critiques au concept social-démocrate et dresse un tableau synthèse (p. 108-109) décrivant les caractéristiques comparées de trois modèles différents de l'État-providence. En conclusion, Groulx écrit que la crise fiscale et économique a ralenti le développement de l'État-providence, a forcé l'intégration des valeurs du marché et $a$, de ce fait, ralenti le progrès des objectifs d'égalité et de redistribution.

On retiendra de cet ouvrage que le modèle suédois, a l'instar de tous les autres nés avec le développerrient économique et industriel, se dirige en droite ligne vers le mur de la réalité, c'est-à-dire la nécessité d'équilibrer le social et l'économique. Malheureusement, l'auteur ne parle pas assez de ce qui est, dans le fond, l'essentiel de la remise en question des divers modèles de l'État-providence. Le réflexe "politically correct" que l'on sent chez Groulx l'empecche d'interroger a fond le modèle suédois et de critiquer les principes fondamentaux de solidarité et d'égalité. II prend pour acquis qu'ils sont incontestables. II a tort. Une réflexion sur ces principes lui aurait permis de sortir l'analyse de son sujet du cadre trop étroit de l'opposition Etat/marché. $L^{\prime}$ 'interrogation universelle sur l'État-providence concorde avec la manifestation du marasme des finances publiques; on en convient aisément. Par contre, la lutte contre les tutelles bureaucratiques est un phénomène culturel qui, lui aussi, se généralise, ce qui amène le citoyen à souhaiter une rénovation de l'État. En somme, si Lionel-Henri Groulx avait été plus audacieux et moins descriptif dans son évaluation, il nous aurait livré un ouvrage beaucoup plus innovateur et plus utile pour la compréhension des politiques publiques.

En conclusion, j'estime que ce livre mérite d'être lu pour son apport documentaire et pour la preuve (tacite) qu'il fournit que la plupart des modèles se ressemblent et qu'ils sont confrontés aux mêmes difficultés.

Pierre P. Tremblay Université du Québec à Montréal 